\title{
G

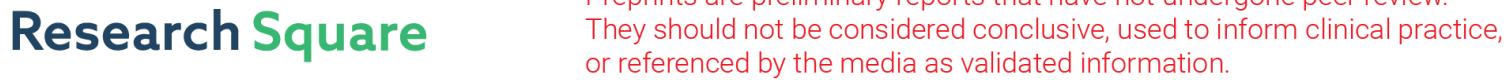 \\ GAS5 Attenuates Malignant Progression of Glioma Stem-like Cells via Regulating E-cadherin
}

\section{Haiyang Wang}

Affiliated Hangzhou First People's Hospital Zhejiang University School of Medicine: Hangzhou First

People's Hospital

\section{Ding Wang}

Affiliated Hangzhou First People's Hospital Zhejiang University School of Medicine: Hangzhou First

People's Hospital

\section{Yongfeng Shen}

Affiliated Hangzhou First People's Hospital Zhejiang University School of Medicine: Hangzhou First

People's Hospital

\section{Chenglong Sun}

Affiliated Hangzhou First People's Hospital Zhejiang University School of Medicine: Hangzhou First

People's Hospital

\section{Qiang Hu}

Affiliated Hangzhou First People's Hospital Zhejiang University School of Medicine: Hangzhou First

People's Hospital

\section{Li Jiang}

Affiliated Hangzhou First People's Hospital Zhejiang University School of Medicine: Hangzhou First

People's Hospital

\section{Quan Du ( $\nabla$ duquan76@zuaa.zju.edu.cn )}

Affiliated Hangzhou First People's Hospital Zhejiang University School of Medicine: Hangzhou First

People's Hospital

\section{Research Article}

Keywords: Glioma stem-like cells (GSCs), LncRNA, GAS5, E-cadherin/CDH1

Posted Date: January 28th, 2022

DOI: https://doi.org/10.21203/rs.3.rs-1177074/v1

License: (c) (i) This work is licensed under a Creative Commons Attribution 4.0 International License. Read Full License 


\section{Abstract}

Background: It is commonly understood that glioma stem-like cells (GSCs) play a vital role in the malignant progression of glioma. Recent studies have reported that long non-coding RNAs (IncRNAs) closely associate with glioma development, however, the underlying molecular regulatory mechanisms on GSCs have not been fully clarified.

Methods: LncRNA GAS5 expression level was analyzed by bioinformatics and qRT-PCR assays. GSCs were separated from glioma tissues and identified using immunofluorescence and flow cytometry. Cell proliferation ability was measured by EdU assays. Cell invasion and migration ability was evaluated by transwell assays. Cell apoptosis was measured by flow cytometry. Subcutaneous tumorigenesis assays were performed to explore the GSCs growth in vivo. Luciferase reporter assays were used to identify the direct molecular interaction. E-cadherin expression level was measured by qRT-PCR assays and western blot.

Results: In this study, we established two highly malignant glioma stem-like cells from clinical surgical specimens, and found that IncRNA GAS5 was downregulated in GSCs and high-grade glioma tissues, and showed a correlation with patient survival. Functional assays showed that depleting GAS5 promoted the proliferation, invasion, migration, stemness and tumorigenicity, and inhibited apoptosis of GSCs. Mechanistically, GAS5 directly sponge with miR-23a which acts as an oncogene by regulating E-cadherin (CDH1). In addition, rescue experiments demonstrated that GAS5 modulate both the expression and function of E-cadherin via the dependent manner of miR-23a.

Conclusions: GAS5 functions as a suppressor in GSCs by targeting the miR-23a/CDH1 axis, which may be a promising therapeutic target against gliomas.

\section{Introduction}

Gliomas are the most commonly occurring primary intracranial tumors in the central nervous system, and glioblastoma multiforme (GBM) is the most prevalent, malignant and aggressive in gliomas ${ }^{1}$. The overall survival and prognosis of GBM are poor, although the therapeutic regimens have been optimizing continuously ${ }^{2}$. Many researchers attribute the poor prognosis to the glioma stem-like cells (GSCs) which harbor remarkable cellular heterogenicity and are responsiable for tumor progression including initiation, metastasis, treatment resistance and so on ${ }^{3}$. But the molecular mechanisms of GSCs have not been elucidated, which may be urgently needed for improving glioma treatment efficacy ${ }^{3,4}$.

Long non-coding RNAs (IncRNAs) have been revealed to play critical roles in various pathophysiological processes via activating or silencing the corresponding on transcription and/or post-transcription level ${ }^{5}$. The increasing evidence supports that IncRNAs are abnormally expressed in various tumors and are involved in multiple malignant phenotypes of tumors ${ }^{6}$. Growth Arrest-Specific 5 (GAS5), located at chromosome 1q25, was frequently downregulated and acted as a potential tumor-suppressor gene in 
various malignancies, such as breast cancer ${ }^{7}$, hepatocellular carcinoma ${ }^{8}$, and ovarian cancer ${ }^{9}$. It has also been reported that GAS5 could curbs the proliferation and metastasis of glioma cells, implicating that it might be a promising threptic treatment of gliomas ${ }^{10}$. However, there has been little study of the function and the related molecular mechanisms of GAS5 in glioma stem-like cells (GSCs), which needs further investigation.

$\mathrm{CDH} 1$ is widely recognized as a tumor suppressor involved in epithelial-to-mesenchymal transition (EMT) and inhibits metastasis, which encodes the cell-cell adhesion protein E-cadherin ${ }^{11}$. EMT enables polarized epithelial cells to mesenchymal cells, exhibiting characteristics of enhanced invasion and migration capacities ${ }^{12}$. The loss of E-cadherin ( $\left.\mathrm{CDH} 1\right)$ expression is the crucial activation factor of EMT, and the downregulation of $\mathrm{CDH} 1$ in glioma contributes to tumor progression ${ }^{13}$.

In the present study, we analyzed the expression of GAS5 in glioma of TCGA and CGGA databases and found GAS5 was negatively associated with the malignancy of glioma. Based on integrated analysis of both function assays and further molecular regulation assays, it was identified that GAS5-mediated $\mathrm{CDH} 1$ suppression via sponging miR-23a inhibited the malignant behavior of GSCs.

\section{Materials And Methods}

\section{Tumor specimens and cell culture}

Clinical cancer and adjacent normal brain tissue specimens derived from glioma patients collected from the Department of Neurosurgery, Affiliated Hangzhou First People's Hospital, Zhejiang University School of Medicine, after obtaining informed consent. The whole process was consistent with the requirement of Ethics Committee of the Zhejiang University School of Medicine.

Primary cultured human GSC1 and GSC2 cells derived from surgical specimen of glioma patients and were cultured in DMEM/F12 medium favored for neural stem cell growth (Gibco, USA) including 1x B27 Supplement (Gibco, US), $20 \mathrm{ng} / \mathrm{ml}$ epidermal growth factor (EGF) (Gibco, US), and $20 \mathrm{ng} / \mathrm{ml}$ basic fibroblast growth factor (bFGF) (Gibco, US). Glioma cell lines (T98G, U251, A172) and normal human astrocyte cells (NHAs) were cultured in DMEM (Hyclone, US) containing 10\% fetal bovine serum (FBS) (BI, Israel). All cells were cultured in the incubator (SANYO, JP) at $37^{\circ} \mathrm{C}$ with $5 \% \mathrm{CO}_{2}$.

\section{Immunocytochemical Staining}

GSCs were fixed with methanol for 20 min at room temperature, permeabilized with $0.25 \%$ Triton X-100 (Beyotime, China), and blocked with blocking buffer for $60 \mathrm{~min}$. The fixed cells were then incubated first with primary antibodies of SOX2, OCT4 (CST, US) for 60 min, respectively. After washing with phosphate buffer saline (PBS) for 3 times, the second antibody (Beyotime, China) was applied for 30 min at room 
temperature in the dark. Finally, the cells were stained with DAPI, and observed under fluorescence microscope (Zeiss, Germany) at a magnification of 200x.

\section{Flow cytometry}

Single-cell suspensions of GSCs were suspended with $100 \mu \mathrm{l}$ of PBS and incubated with $5 \mu \mathrm{l}$ primary antibodies of anti-SOX2 and anti-OCT4 (dilution 1:200) for $1 \mathrm{~h}$ at room temperature, after digestion and centrifugation. Then the cells were washed with PBS, and incubated with secondary antibody (Beyotime, China) for $1 \mathrm{~h}$ at room temperature. After centrifugation and washes with PBS, the cells were resuspended in $200 \mu \mathrm{l}$ of PBS and finally analyzed with flow cytometry with Cytexpert 2.0 software.

\section{Vector construction and cell transfection}

The overexpression vector of GAS5, the short hairpin RNA (shRNA) targeting GAS5 (shGAS5-1 and shGAS5-2) and the corresponding negative control, the miR-23a mimics, inhibitors, and corresponding negative control, were all constructed by GenePharma (Shanghai, China). The vectors and corresponding controls were transfected into GSCs according to the manufacturer's protocol.

\section{Quantitative real-time reverse transcription PCR (qRT-PCR)}

Total RNA was extracted from cells with TRIzol (Invitrogen, US) and reverse transcribed to cDNA using the reverse transcription kit (Thermo, US). qRT-PCR was performed to measure the expression levels of GAS5, $\mathrm{CDH} 1$ and miR-23a. The expression of GAPDH and U6 served as control. The expression level was analyzed by $2^{-\triangle \Delta C t}$ method.

\section{5-Ethynyl-20-deoxyuridine (EdU) assay}

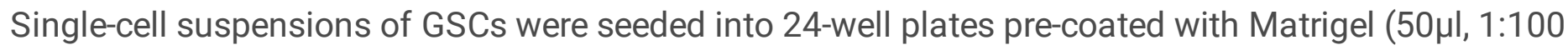
dilution, $\mathrm{BD}, \mathrm{US}$ ) for adherence in an incubator containing $5 \% \mathrm{CO}_{2}$ at $37^{\circ} \mathrm{C} .50 \mu \mathrm{M} \mathrm{EdU} \mathrm{(RiboBio,} \mathrm{China)}$ was added to each well for incubation $2 \mathrm{~h}$. Then the cells were fixed in $4 \%$ polyformaldehyde for 20 min and stained by Apollo dye solution for $25 \mathrm{~min}$. Cell nuclei were stained with DAPI for $20 \mathrm{~min}$. The proportion of EdU-positive cells were analyzed under a fluorescence microscope.

\section{Transwell assay}

Transwell assays were performed to evaluate the invasion and migration of GSCs. Some upper chambers

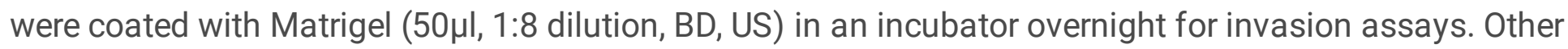




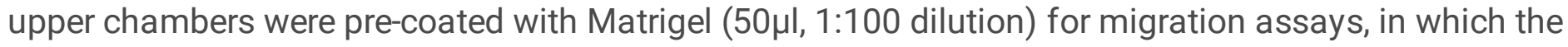
Matrigel was aspirated after $20 \mathrm{~min}$. The subsequent protocol was performed as previously described ${ }^{14}$.

\section{GSCs sphere formation assay}

Single-cell suspensions of GSCs were seeded on 24-well plates at a density of 100 cells per well. The sphere numbers of GSCs $(>50 \mu \mathrm{m})$ in each well was counted under a microscope. The sphere formation efficiency (SFE) of GSCs was calculated by the spheres numbers $/ 100 \times 100 \%$ method.

\section{Cell apoptosis assay}

The cell apoptosis rate of GSCs was detected by the kit of annexin V-APC/7-AAD (BD, US). Single-cell suspensions of GSCs were washed by PBS and re-suspended by binding buffer including. $5 \mu$ l annexin VAPC and $5 \mu$ l 7-AAD were added to the suspensions of GSCs (15 min each), respectively, in the dark at room temperature. The cell apoptosis rate was analyzed by flow cytometry within $1 \mathrm{~h}$ after staining.

\section{Tumorigenicity assay}

4-6 weeks old athymic Balb/c nude mice (15-20 g) were bred in the animal center under specific pathogen-free (SPF) conditions. Single cell suspensions of $1 \times 10^{6} \mathrm{GSCs}$ with up/downregulation of GAS5 and the corresponding negative control were injected subcutaneously into the right flank of each mouse, respectively. 3 weeks later, the volume and weight of the tumors were calculated by length $\times$ width ${ }^{2} \times 0.5$ ).

\section{Luciferase reporter assay}

The wild-type and mutant fragments with miR-23a binding sequences in the 3 '-untranslated region (UTR) of GAS5 and CDH1 were inserted into pMIR-REPORT vectors. Then the PMIR-REPORT vectors, together with miR-23a mimics or the corresponding negative control, were transfected into GSCs for $48 \mathrm{~h}$. The dualluciferase reporter assay system (Promega, US) was used to evaluate the luciferase activity of GSCs.

\section{Western blotting}

Total protein from GSCs was extracted by RIPA buffer (Beyotime, China). Protein concentration was determined by the BCA Protein Assay Kit (Beyotime, China), after which $20 \mu \mathrm{g}$ total protein were separated by $10 \%$ SDS-PAGE, transferred to PVDF membrane, and then incubated with primary antibodies against $\mathrm{CDH} 1$ (CST, US) and GAPDH (CST, US) overnight at $4^{\circ} \mathrm{C}$. The membranes were then incubated with secondary antibody for $60 \mathrm{~min}$ at room temperature. Enhanced chemiluminescence (ECL) method was used for visualization for quantitative analysis. 


\section{Statistical analysis}

All data in this study were presented as mean $\pm S D$ and analysed with the GraphPad Prism software (Version 8.0.2, (CA, USA). One-way/two-way analysis of variance (one/two-way ANOVA) was used to determine the differences among at least three groups. Student's $t$ test was used to evaluate the differences between two groups. $P$ value $<0.05$ was accepted as statistically significant $\left({ }^{*} p<0.05 ; * \star p<\right.$ $0.01 ; * \star * p<0.001 ; \star \star \star \star ~ p<0.0001)$.

\section{Results}

1. The expression of GAS5 was negatively correlated with the malignancy of glioma.

The expression of GAS5 was analyzed in different subtype of glioma in TCGA and CGGA database, we found that the GAS5 expression was negatively correlated with the malignancy and overall survival of glioma in databases of CGGA and TCGA. Based on IDH mutation status classification of glioma, remarkable differences of GAS5 expression between IDH mutant subtype and IDH wildtype subtype were also analyzed. The expression of GAS5 in IDH wildtype subtype was significantly decreased compared with IDH mutant subtype in CGGA_693 datasets and TCGA_GBMLGG datasets (Fig. 1A, B). The similar expression profile showed that the expression of GAS5 in 1p/19q non-codeleted subtype was significantly lower than that of 1p/19q codeleted subtype (Fig. 1C, D). Further analysis found that the expression of GAS5 in IDH wildtype subtype was significantly lower than that of IDH mutant combined with $1 p / 19 q$ codeleted subtype and IDH mutant combined with $1 p / 19 q$ non-codeleted subtype (Fig. 1E, F). Overall survival analysis of gliomas showed that the survival rate of patients with low GAS5 expression decreased significantly (Fig. 1G, H).

\section{Primary culture of GSCs derived from clinical surgical specimens}

To investigate the function of GAS5 in GSCs, we cultured two human GSCs (GSC1 and GSC2), which respectively derived from two patients diagnosed glioblastoma. The CSC1 and GSC2 not only exhibited typical sphere-like cell clusters, but also could adherently grow on laminin coated plates (Fig. 2A). To further verify the cells were GSCs, we detected the GSCs makers of GSC1 and GSC2. Immunofluorescence assays indicated that the GSCs makers (SOX2 and OCT4) were positive in GSC1 and GSC2 (Fig. 2B). And flow cytometric analysis showed the positivity rates of the GSC markers (SOX2 and OCT4) in GSC1 and GSC2 were all more than $70 \%$ (Fig. 2C).

3. GAS5 upregulation inhibited proliferation, invasion, migration and stemness, and promoted apoptosis of GSCs in vitro.

GAS5 expression was further detected in the GSCs (GSC1 and GSC2), glioma cell lines (T98G, U251, A172), and glioma tissue, which disclosed GAS5 downregulation in GSCs and glioma cells and tissue (Fig. 3A, B). To evaluate the function of GAS5 in GSCs, GSC1 and GSC2 were transfected with GAS5 overexpression plasmid and the corresponding negative control, the transfection efficiency was validated 
by qRT-PCR (Fig. 2C). EdU assays indicated that GAS5 upregulation decreased the proliferation ability of GSC1 and GSC2 cells (Fig. 2D, E). Transwell assays showed that overexpression of GAS5 resulted in significant decline of invasion and migration ability of GSC1 and GSC2 cells (Fig. 2F-I). And sphere formation assays indicated that upregulation of GAS5 remarkably weakened the sphere formation efficiency of GSC1 and GSC2 cells (Fig. 2J-K). Furthermore, flow cytometric assays verified that GAS5 upregulation in GSC1 and GSC2 cells led to obvious increase in cell apoptosis rate (Fig. 2L-M).

4. Silencing GAS5 promoted proliferation, invasion, migration and stemness, and inhibited apoptosis of GSCs in vitro.

To further verify the function of GAS5 in GSCs, silencing GAS5 expression in GSC1 and GSC2 cells was achieved via transfection of short hairpin RNAs (shRNAs) targeting GAS5 (including shGAS5-1 and shGAS5-2) and the corresponding negative control, and verified by qRT-PCR (Fig. 4A). EdU assays showed GAS5 downregulation enhanced the proliferation capacity of GSC1 and GSC2 cells (Fig. 4B-D). The results of Transwell assays indicated that silencing GAS5 expression promoted the invasion and migration of GSC1 and GSC2 cells (Figures 4E-H). Sphere formation assays suggested that downregulation of GAS5 significantly increased the sphere formation efficiency of GSC1 and GSC2 cells (Fig. $2 \mathrm{l}, \mathrm{J}$ ). And flow cytometric assays suggested that GAS5 downregulation in GSC1 and GSC2 cells led to increase in cell apoptosis rate (Fig. 2K, L).

5. GAS5 inhibited the growth of GSCs in vivo.

To evaluate the effect of GAS5 on GSCs growth in vivo, tumorigenicity assays were performed. Subcutaneous injection of GSC1 cells transfected with shGAS5 or the corresponding negative control was performed, and the results showed that GAS5 downregulation of GSCs led to increase in both tumor volume and weight, compared with the control group (Fig. 5A-C). In addition, GSC2 cells transfected with TUG overexpression vector or the corresponding negative control were subcutaneous implanted, and the results showed that overexpression of GAS5 in GSCs led to lower tumor volume and weight, compared with the control group (Fig. 5D-F).

\section{GAS5 acted as a sponge for miR-23a in GSCs}

To explore the mechanisms on regulating biological function of GSCs, bioinformatics analysis was applied to identify the possible miRNA targets of GAS5. Potential bonding sites between GAS5 and miR23a were predicted via the online database StarBase (Fig. 6A). Further verification with qRT-PCR found upregulation expression of miR-23a in GSCs, glioma cell lines and glioma tissue, compared with normal human astrocytes (NHAs) and normal brain tissue (Fig. 6B, C). Specifically, miR-23a was negatively regulated by GAS5 in GSCs (Fig. 6D, E). To clarify the direct interaction between miR-23a and GAS5, wild type (WT) and mutant type (MUT) vector of GAS5 were constructed for luciferase activity assays, which showed that miR-23a significantly inhibited the luciferase activity of GAS5-WT (Figures 6F, G), indicating that miR-23a was the direct target of GAS5. 
7. Silencing miR-23a inhibited the proliferation, invasion and migration, and promoted apoptosis of GSCs by targeting $\mathrm{CDH} 1$.

According to bioinformatic analysis, $\mathrm{CDH} 1$ was one of the putative downstream targets of miR-23a (Figures 7A). And to further validate the direct bonding between miR-23a and CDH1, CDH1-WT and CDH1MUT vectors were constructed for luciferase activity assays (Figure 7A). To further explore the regulatory relationship between miR-23a and $\mathrm{CDH} 1$, both of miR-23a inhibitor and $\mathrm{CDH} 1$ shRNA were transfected into GSC $1 / 2$ cells. The assays of qRT-PCR and western blot indicated that miR-23a downregulation can increase $\mathrm{CDH} 1$ expression, and this effect can be partially offset by shRNA transfection of CDH1 (Fig. 7BD). And luciferase reporter assays suggested that miR-23a significantly decreased the luciferase activity of CDH1-WT, compared with CDH1-MUT (Fig. 7E, F). Furthermore, EdU assays showed silencing miR-23a weakened the proliferation of GSCs, and the function can be reversed by downregulation of CDH1 (Fig. 7G-I). Transwell assays indicated that the weakened invasion and migration ability of GSCs induced by silencing miR-23a were partially offset by downregulation of $\mathrm{CDH} 1$ (Fig. 7J-M). And flow cytometric assays suggested that reduced miR-23a in GSCs led to increase in cell apoptosis rate, which can be reversed by downregulation of $\mathrm{CDH} 1$ (Fig. $7 \mathrm{~N}, 0$ ).

8. Silencing GAS5 promoted the proliferation, invasion and migration, and inhibited apoptosis of GSCs by targeting miR-23a.

To elucidate the mechanism which GAS5 downregulation promoted malignancy of GSCs via regulating miR-23a/CDH1 axis, GSC1 and GSC2 cells were transfected with shGAS5 or shGAS5 together with miR23a inhibitors. qRT-PCR and western blot assays suggested that shGAS5 can inhibit CDH1 expression, which was partly offset by miR-23a inhibitors (Fig. 8A-C). Besides, EdU assays indicated shGAS5 enhanced the proliferation capacity of GSCs, which could be reversed by miR-23a inhibitors (Fig. 8D-F). Consistent with results of EdU assays, the increased ability of invasion and migration of GSCs induced by shGAS5 were partly rescued by miR-23a inhibitors as well (Fig. 8G-J). Flow cytometric analysis suggested that shGAS5 inhibited the apoptosis level of GSCs, which could be reversed by miR-23a inhibitors (Fig. 8K-L).

\section{Discussion}

Glioblastoma multiforme (GBM) is one of the most aggressive and common primary tumor in central nervous system, although comprehensive therapies have been improving ${ }^{15}$. within the bulk tumor, glioma stem cells (GSCs) exist as a minor subpopulation, but actively contribute to dismal prognosis including tumor recurrence and chemo- and radio-therapy resistance, which can self-renew and flourish in an unfavorable tumor microenvironment ${ }^{16}$. GSCs not only exhibit potent tumor-initiating or tumorpropagating characteristics but also promote malignant invasion ${ }^{17}$. In consequence, therapies targeting GSCs have been crucial for improving GBM treatment and overcoming therapeutic resistance ${ }^{18}$. In the present study, we constructed two GSCs, derived from clinical specimens of glioma patients, and 
identified the marker of neural stem cells, to investigate the crucial therapeutic target gene of GSCs which was more closer to the real conditions.

Recently, an increasing number of studies has suggested that IncRNAs play an important role in the occurrence and progression of gliomas ${ }^{19}$. And investigating the molecular regulation mechanisms of IncRNAs may provide a promising therapeutic target in gliomas. Growth arrest-specific 5 (GAS5), located on chromosome 1q25, plays an anti-oncogene role in tumors. Low expression of GAS5 is associated with poor survival and cisplatin resistance in cervical cancer through regulation of PDCD $4{ }^{20}$. In addition, GAS5 can enhance radio-sensitivity of esophageal squamous cell carcinoma by upregulating RECK ${ }^{21}$. It is also reported that GAS5 acts as a molecular switch for proliferation regulation in CD133+ cells via inhibiting glucocorticoid receptor (GR) mediated cell cycle control in pancreatic cancer ${ }^{22}$. Moreover, lung cancer cell derived exsomal GAS5 was regard as a biomarker to identify early-stage in non-small cell lung cancer ${ }^{23}$. It was also reported that GAS5 could effectively inhibit the growth and invasion capacity of glioma cells via targeting GSTM ${ }^{24}$. However, the function and relative mechanisms of GAS5 in GSCs remains unclear up to now. In our experiments, we confirmed the GAS5 efficiently inhibit the proliferation, invasion, migration and tumorgenicity, and promote apoptosis of GSCs through regulating miR-23a/CDH1.

miRNAs have recently emerged as key players in tumor progression. MiR-23a is associated with the regulation of various cellular processes, including cell proliferation, apoptosis and metastasis, and also play crucial roles in several types of tumors with different effects. It was reported that miR-23a was upregulated and could promote proliferation and suppress apoptosis by targeting PDCD4 in gastric cancer $^{25}$. The upregulation of miR-23a also promotes esophageal squamous cell carcinoma through targeting TRAF $5^{26}$. In contrast, miR-23a was downregulated and functioned as a anti-oncogene in breast cancer $^{27}$. It was also found that miR-23a could promote progression in glioma via suppressing PTEN ${ }^{28}$. However, the role of miR-23a played in GSCs has never been reported previously. According to our research, down-regulated miR-23a can inhibit the proliferation, invasion, migration, and promote apoptosis of GSCs via targeting CDH1.

$\mathrm{CDH} 1$, a crucial component in EMT, is widely known as a pivotal protein for holding epithelial cells tight by maintaining cell to cell junctions ${ }^{29}$. Downregulation of $\mathrm{CDH} 1$ could decrease the cellular adhesion and promoted the invasion and metastatic of tumor ${ }^{30}$. It was reported that BMP4 suppressed cell invasion of GBM through upregulation of $\mathrm{CDH}^{31}$. And Univariate Cox regression analysis showed that low expression of $\mathrm{CDH} 1$ was associated with shorter progression-free survival in ependymoma ${ }^{32}$. The results of our experiments suggested that the expression of $\mathrm{CDH} 1$ was promoted by GAS5 via sponging miR23a.

\section{Conclusions}

In summary, our study not only uncovered the crucial effects of GAS5 as a tumor suppressor in GSCs but also explored the molecular regulation mechanisms through which miR-23a contributed to the 
progression of GSCs and identified CDH1 (E-cadherin) as a direct target, which might provide new experimental evidence for potential treatment strategy targeting on GSCs in glioma.

\section{Declarations}

\section{Ethics approval and consent to participate:}

The study involving human participants were reviewed and approved by Ethics Committee of the Zhejiang University School of Medicine.

\section{Consent for publication:}

The patients/participants provided written informed consent to participate in this study, and had consent for publication.

\section{Availability of data and materials:}

The datasets generated during and analysed during the current study are available in the TCGA and CGGA repository, [https://portal.gdc.cancer.gov; http://cgga.org.cn/index.jsp]

\section{Competing interests:}

No conflict of interest exists in the submission of the manuscript which is approved by all authors for publication.

\section{Funding:}

Agricultural and social development project of Hangzhou (NO. 2020ZDSJ0900)

\section{Authors' contributions:}

QD is responsible for the design of the study; HW is responsible for experimental implementation; DW is responsible for collection and analysis of data; YS and CS are responsible for the writing the manuscript; $\mathrm{QH}$ and $\mathrm{LJ}$ are responsible for reviewing the manuscript. All authors have read and approved the manuscript.

\section{Acknowledgement:}

Not applicable

\section{References}

1. Liu, Y. et al. Sphingosine 1-Phosphate Liposomes for Targeted Nitric Oxide Delivery to Mediate Anticancer Effects against Brain Glioma Tumors. Advanced materials (Deerfield Beach, Fla.) 33, e2101701, doi:10.1002/adma.202101701 (2021). 
2. Wang, D. et al. CRISPR Screening of CAR T Cells and Cancer Stem Cells Reveals Critical Dependencies for Cell-Based Therapies. Cancer Discov 11, 1192-1211, doi:10.1158/2159-8290.CD20-1243 (2021).

3. Taga, T. \& Tabu, K. Glioma progression and recurrence involving maintenance and expansion strategies of glioma stem cells by organizing self-advantageous niche microenvironments. Inflamm Regen 40, 33, doi:10.1186/s41232-020-00142-7 (2020).

4. Li, J. et al. Hypoxic Glioma Stem Cell-Derived Exosomes Containing Linc01060 Promote Progression of Glioma by Regulating the MZF1/c-Myc/HIF1alpha Axis. Cancer Res 81, 114-128, doi:10.1158/0008-5472.CAN-20-2270 (2021).

5. Luo, M. L. et al. The Role of APAL/ST8SIA6-AS1 IncRNA in PLK1 Activation and Mitotic Catastrophe of Tumor Cells. J Natl Cancer Inst 112, 356-368, doi:10.1093/jnci/djz134 (2020).

6. Tan, Y. T. et al. LncRNA-mediated posttranslational modifications and reprogramming of energy metabolism in cancer. Cancer Commun (Lond) 41, 109-120, doi:10.1002/cac2.12108 (2021).

7. Li, G. et al. Long noncoding RNA growth arrestspecific 5 (GAS5) acts as a tumor suppressor by promoting autophagy in breast cancer. Mol Med Rep 22, 2460-2468, doi:10.3892/mmr.2020.11334 (2020).

8. Zhang, W. Y. et al. Long noncoding RNA Gas5 induces cell apoptosis and inhibits tumor growth via activating the $\mathrm{CHOP}$-dependent endoplasmic reticulum stress pathway in human hepatoblastoma HepG2 cells. J Cell Biochem, doi:10.1002/jcb.30159 (2021).

9. Long, X. et al. Long non-coding RNA GAS5 inhibits DDP-resistance and tumor progression of epithelial ovarian cancer via GAS5-E2F4-PARP1-MAPK axis. J Exp Clin Cancer Res 38, 345, doi:10.1186/s13046-019-1329-2 (2019).

10. Ding, Y., Wang, J., Zhang, H. \& Li, H. Long noncoding RNA-GAS5 attenuates progression of glioma by eliminating microRNA-10b and Sirtuin 1 in U251 and A172 cells. Biofactors 46, 487-496, doi:10.1002/biof.1604 (2020).

11. Mendonsa, A. M., Na, T. Y. \& Gumbiner, B. M. E-cadherin in contact inhibition and cancer. Oncogene 37, 4769-4780, doi:10.1038/s41388-018-0304-2 (2018).

12. Pastushenko, I. \& Blanpain, C. EMT Transition States during Tumor Progression and Metastasis. Trends Cell Biol 29, 212-226, doi:10.1016/j.tcb.2018.12.001 (2019).

13. Tao, C. et al. Genomics and Prognosis Analysis of Epithelial-Mesenchymal Transition in Glioma. Front Oncol 10, 183, doi:10.3389/fonc.2020.00183 (2020).

14. Wang, H. et al. HOTAIRM1 Promotes Malignant Progression of Transformed Fibroblasts in Glioma Stem-Like Cells Remodeled Microenvironment via Regulating miR-133b-3p/TGFbeta Axis. Front Oncol 11, 603128, doi:10.3389/fonc.2021.603128 (2021).

15. Alafate, W. et al. Loss of PLK2 induces acquired resistance to temozolomide in GBM via activation of notch signaling. J Exp Clin Cancer Res 39, 239, doi:10.1186/s13046-020-01750-4 (2020).

16. Lah, T. T., Novak, M. \& Breznik, B. Brain malignancies: Glioblastoma and brain metastases. Semin Cancer Biol 60, 262-273, doi:10.1016/j.semcancer.2019.10.010 (2020). 
17. Boyd, N. H. et al. Glioma stem cells and their roles within the hypoxic tumor microenvironment. Theranostics 11, 665-683, doi:10.7150/thno.41692 (2021).

18. Hou, Y. et al. Targeting of glioma stem-like cells with a parthenolide derivative ACT001 through inhibition of AEBP1/PI3K/AKT signaling. Theranostics 11, 555-566, doi:10.7150/thno.49250 (2021).

19. Peng, Z., Liu, C. \& Wu, M. New insights into long noncoding RNAs and their roles in glioma. Mol Cancer 17, 61, doi:10.1186/s12943-018-0812-2 (2018).

20. Fang, X. et al. Low GAS5 expression may predict poor survival and cisplatin resistance in cervical cancer. Cell Death Dis 11, 531, doi:10.1038/s41419-020-2735-2 (2020).

21. Lin, J. et al. Elevation of long non-coding RNA GAS5 and knockdown of microRNA-21 up-regulate RECK expression to enhance esophageal squamous cell carcinoma cell radio-sensitivity after radiotherapy. Genomics 112, 2173-2185, doi:10.1016/j.ygeno.2019.12.013 (2020).

22. Sharma, N. S. et al. Long non-coding RNA GAS5 acts as proliferation "brakes" in CD133+ cells responsible for tumor recurrence. Oncogenesis 8, 68, doi:10.1038/s41389-019-0177-4 (2019).

23. Li, C. et al. Tumor-derived exosomal IncRNA GAS5 as a biomarker for early-stage non-small-cell lung cancer diagnosis. J Cell Physio/ 234, 20721-20727, doi:10.1002/jcp.28678 (2019).

24. Li, G., Cai, Y., Wang, C., Huang, M. \& Chen, J. LncRNA GAS5 regulates the proliferation, migration, invasion and apoptosis of brain glioma cells through targeting GSTM3 expression. The effect of LncRNA GAS5 on glioma cells. J Neurooncol 143, 525-536, doi:10.1007/s11060-019-03185-0 (2019).

25. $\mathrm{Hu}, \mathrm{X}$. et al. miR-23a/b promote tumor growth and suppress apoptosis by targeting PDCD4 in gastric cancer. Cell Death Dis 8, e3059, doi:10.1038/cddis.2017.447 (2017).

26. Shang, M. et al. HP1BP3 promotes tumor growth and metastasis by upregulating miR-23a to target TRAF5 in esophageal squamous cell carcinoma. Am J Cancer Res 11, 2928-2943 (2021).

27. Zhu, L. et al. IncRNA NEAT1 promotes the Taxol resistance of breast cancer via sponging the miR23a-3p-FOXA1 axis. Acta Biochim Biophys Sin (Shanghai) 53, 1198-1206, doi:10.1093/abbs/gmab098 (2021).

28. He, Z. et al. LncRNA DGCR5 plays a tumor-suppressive role in glioma via the miR-21/Smad7 and miR-23a/PTEN axes. Aging (Albany NY) 12, 20285-20307, doi:10.18632/aging.103800 (2020).

29. Lin, Y. T. \& Wu, K. J. Epigenetic regulation of epithelial-mesenchymal transition: focusing on hypoxia and TGF-beta signaling. J Biomed Sci 27, 39, doi:10.1186/s12929-020-00632-3 (2020).

30. Vos, E. L. et al. Indications for Total Gastrectomy in CDH1 Mutation Carriers and Outcomes of RiskReducing Minimally Invasive and Open Gastrectomies. JAMA Surg 155, 1050-1057, doi:10.1001/jamasurg.2020.3356 (2020).

31. Zhao, X., Sun, Q., Dou, C., Chen, Q. \& Liu, B. BMP4 inhibits glioblastoma invasion by promoting Ecadherin and claudin expression. Front Biosci (Landmark Ed) 24, 1060-1070, doi:10.2741/4768 (2019).

32. Malgulwar, P. B. et al. Epithelial-to-mesenchymal transition-related transcription factors are upregulated in ependymomas and correlate with a poor prognosis. Hum Pathol 82, 149-157, 
doi:10.1016/j.humpath.2018.07.018 (2018).

Figures
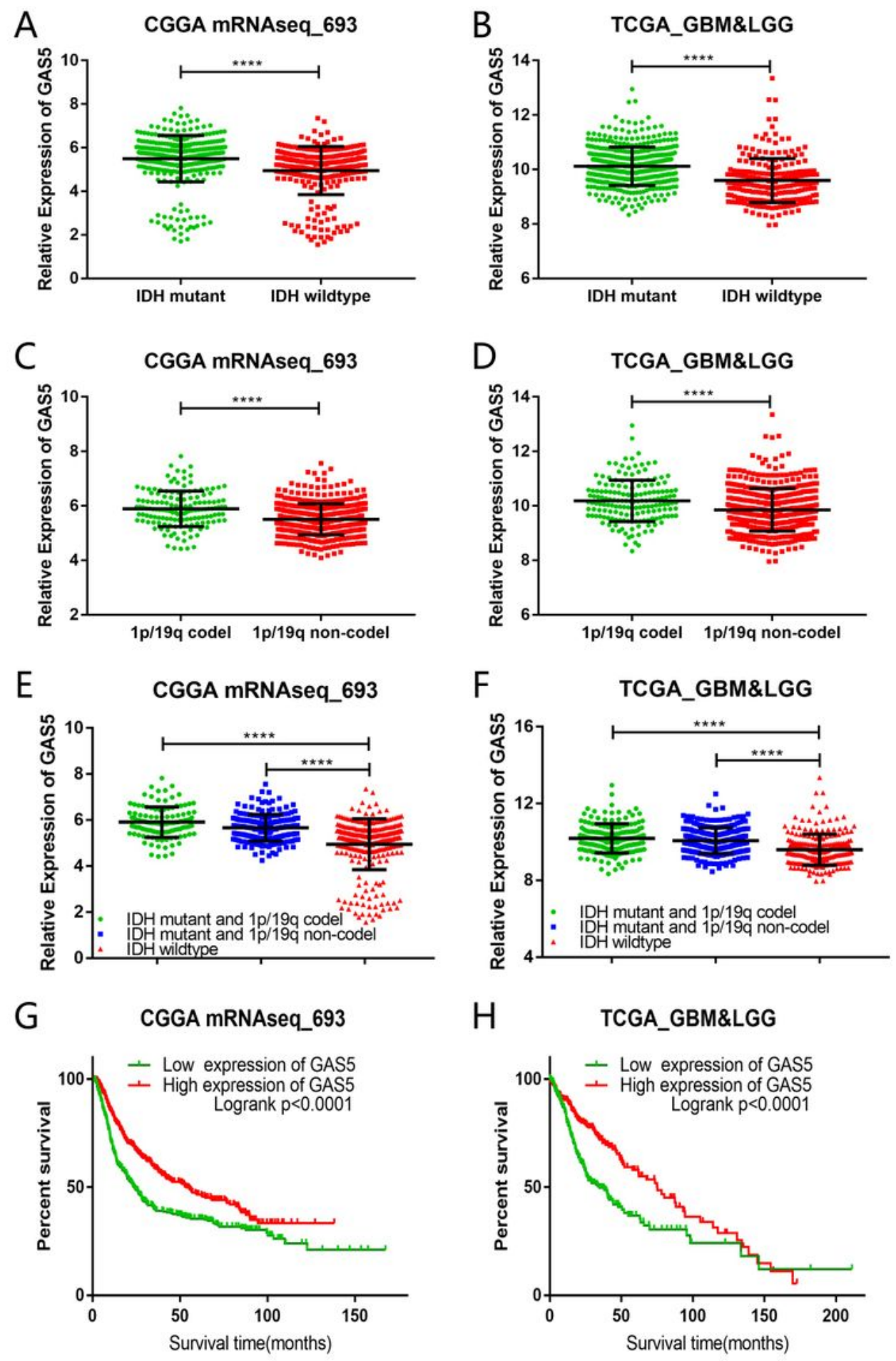

Figure 1 
(A, B) The difference expression of GAS5 between IDH mutant and IDH wildtype subtypes in CGGA_693 and TCGA_GBMLGG datasets, respectively. $* \star \star \star p<<0.0001$, Student's $t$ test. (C, D) The difference of GAS5 expression between 1p/19q codeleted and 1p/19q non-codeleted subtypes in in CGGA_693 and TCGA_GBMLGG datasets, respectively. ${ }^{* \star \star} \mathrm{p}<0.0001$, Student's $t$ test. $(\mathrm{E}, \mathrm{F})$ The difference of GAS5 expression among IDH mutant and 1p/19q codeleted, IDH mutant and 1p/19q non-codeleted, and IDH wildtype subtypes in CGGA_693 and TCGA_GBMLGG datasets, respectively. ${ }^{\star \star \star \star} p<0.0001$, one-way ANOVA. $(G, H)$ Overall survival rate of glioma patients in low GAS5 group and high GAS5 group.
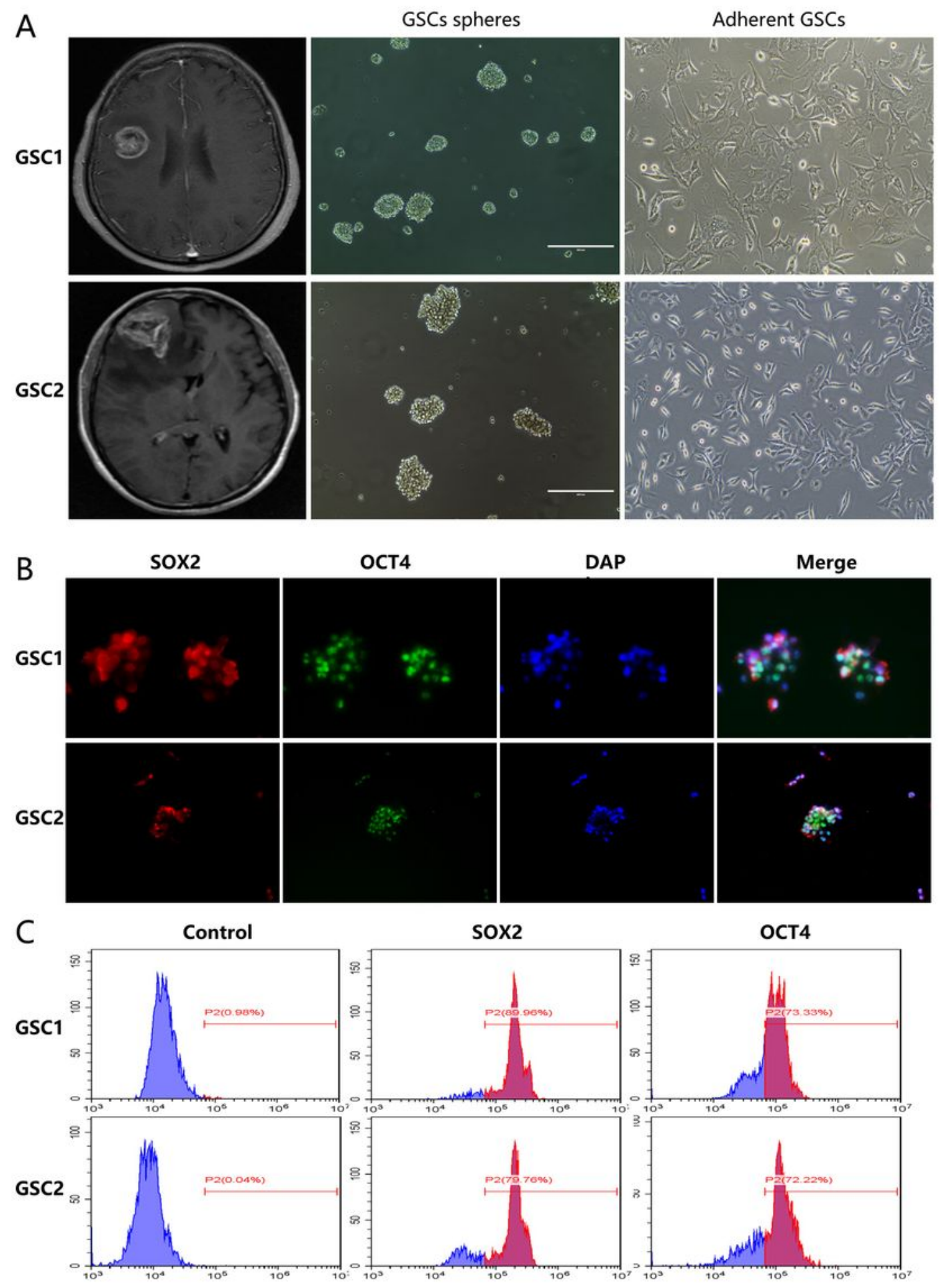
Figure 2

(A) Primary culture of human GSCs derived from two patients diagnosed glioblastoma. Sphere-like cell clusters and adherent growth of GSCs were cultured in medium designed to support stem cell growth. (B) The GSCs makers were detected by Immunofluorescence assays in GSC1 and GSC2. (C) The positivity rates of the GSC markers were analyzed by flow cytometric in GSC1 and GSC2.

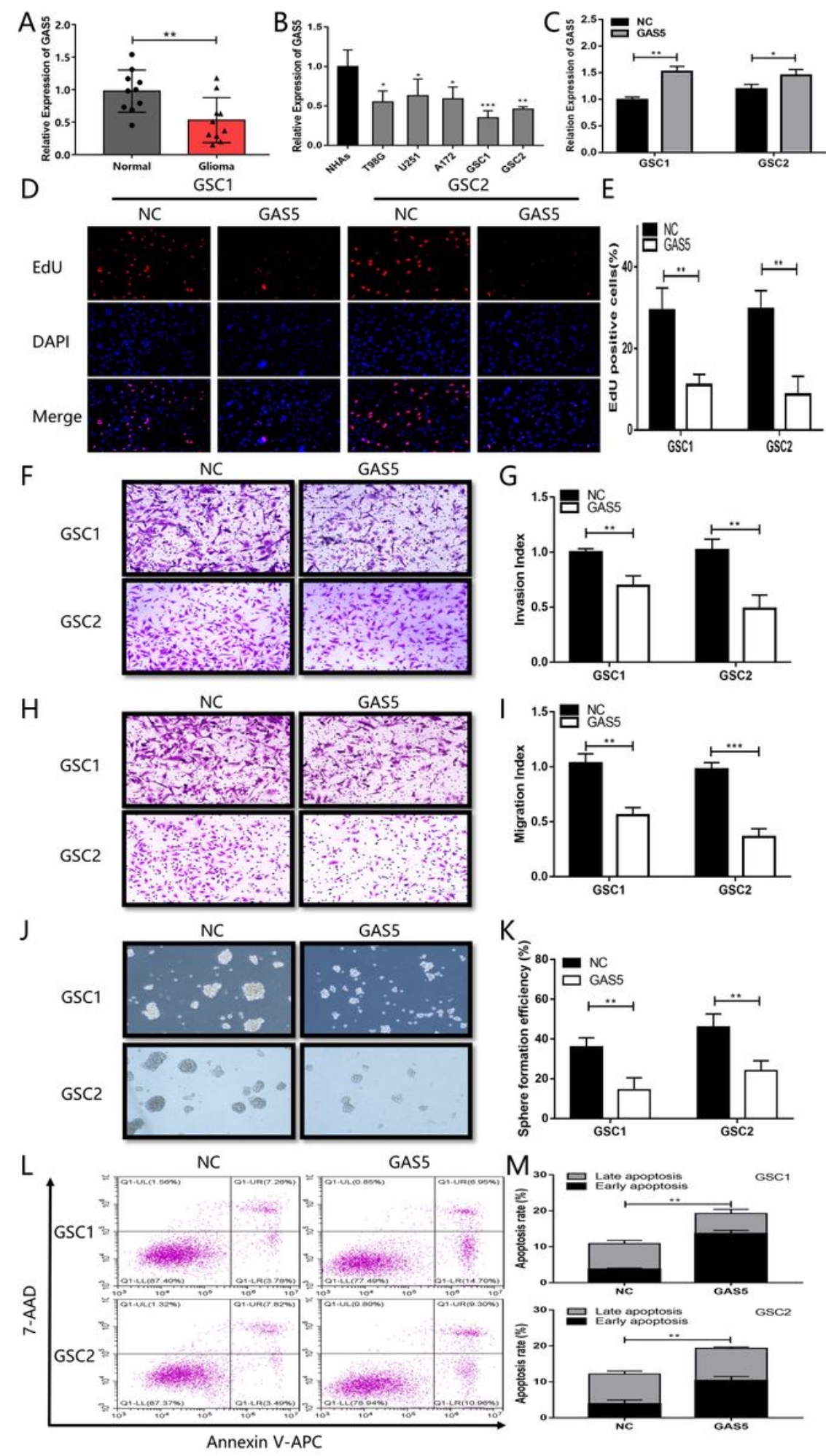




\section{Figure 3}

(A, B) GAS5 expression was analyzed by qRT-PCR in glioma tissue, glioma cell lines and GSCs. (C) GAS5 expression was analyzed with qRT-PCR in GSCs transfected with GAS5 or NC. *p $<0.05$, ${ }^{\star *} p<0.01$, Student's $t$ test. (D, E) Proliferation ability was evaluated after GAS5 upregulation in GSCs using EdU assays. ${ }^{*} p<0.01$, Student's $t$ test. $(F, I)$ Invasion and migration capacity was assessed after GAS5 upregulation in GSCs by transwell assays. ${ }^{\star \star} p<0.01,{ }^{\star \star \star} p<0.001$, Student's $t$ test. $(\mathrm{J}, \mathrm{K})$ The stemness was evaluated after GAS5 upregulation in GSCs by sphere formation assays. (L, M) Cell appotosis was evaluated via flow cytometric assays after GAS5 upregulation in GSCs. 


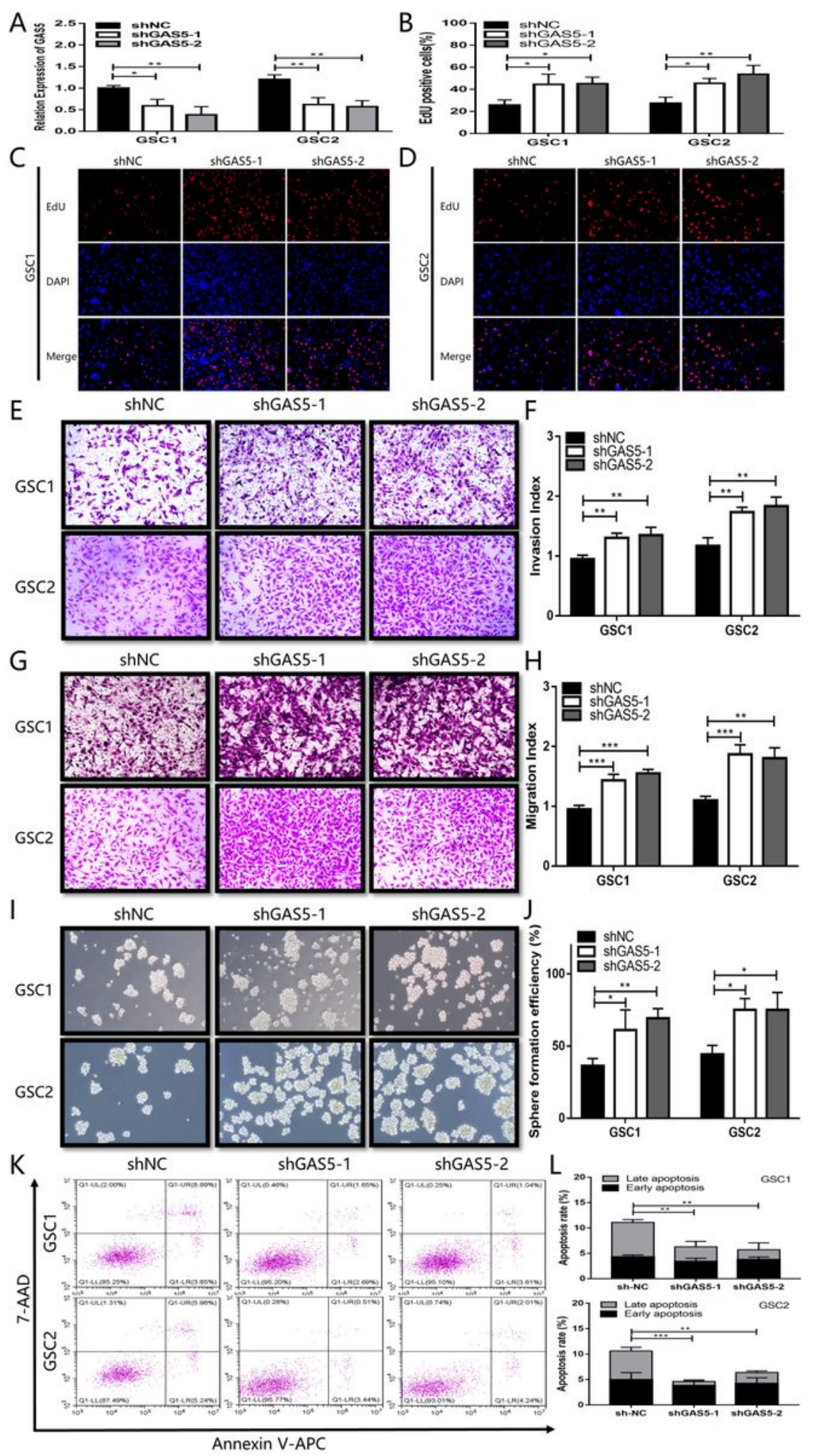

Figure 4

(A) qRT-PCR analysis of GAS5 expression in GSCs after transfection of the shNC, shGAS5-1 or shGAS5-2. ${ }^{*} \mathrm{p}<0.05,{ }^{*} \mathrm{p}<0.01$, one-way ANOVA. (B-D) Proliferation ability was evaluated after GAS5 downregulation in GSCs using EdU assays. ${ }^{\star} p<0.05$, ${ }^{*} \mathrm{p}<0.01$, one-way ANOVA. (E-H) Cell invasion and migration capacity were measured after GAS5 downregulation in GSCs by transwell assays. ${ }^{* *} p<0.01$, $\star * \star \mathrm{p}<0.001$, one-way ANOVA. $(\mathrm{I}, \mathrm{J})(\mathrm{J}, \mathrm{K})$ The stemness was evaluated after GAS5 downregulation in 
GSCs by sphere formation assays. (K, L) Cell appotosis was evaluated via flow cytometric assays after GAS5 downregulation in GSCs.
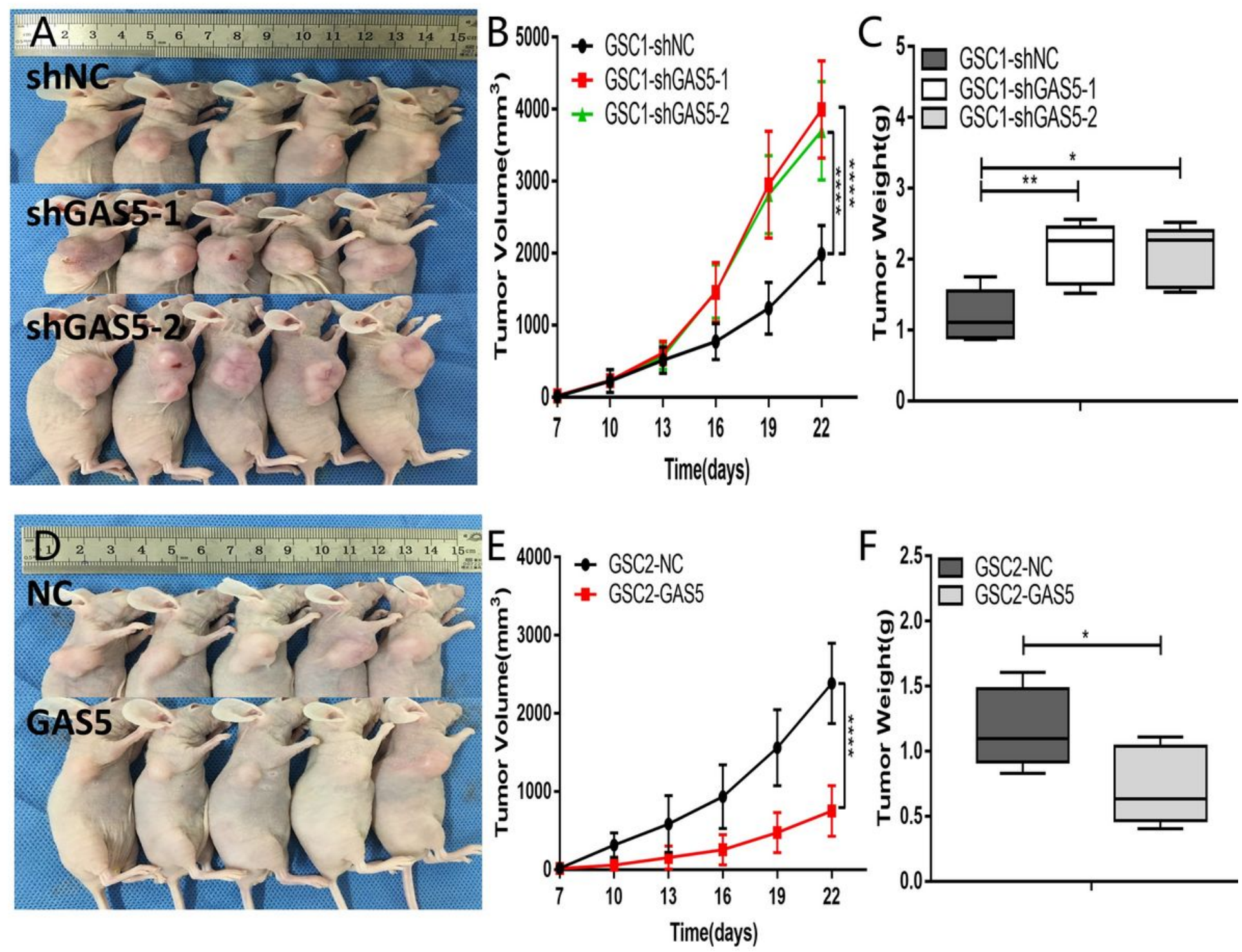

Figure 5

(A) Tumorigenicity assays performed in nude mice by subcutaneous inoculation of GSC1 cells transfected with shNC, shGAS5-1, or shGAS5-2, respectively. (B, C) Tumor growth curve and tumor weight of shNC, shGAS5-1, or shGAS5-2 group. ${ }^{* \star *} \mathrm{p}<0.0001$, two-way ANOVA; ${ }^{*} p<0.05,{ }^{*} \mathrm{p}<0.01$, one-way ANOVA. (D) Tumorigenicity assays performed in nude mice by subcutaneous inoculation of GSC2 cells transfected with NC or GAS5, respectively. (E, F) Tumor growth curve and tumor weight of NC or GAS5 group. ${ }^{* \star \star} p<0.0001$, two-way ANOVA; ${ }^{\star} p<0.05$, Student's $t$ test. 
A

miR-23a

3'-ccuuuagggaccgUUACACUa-5'

| | | | | | |

GAS5 WT 5'-uuuaauacugaagaaAAUGUGAa-3'

GAS5 MUT 5'-uuuaauacugaagaaAAUGUGAa-3'

B

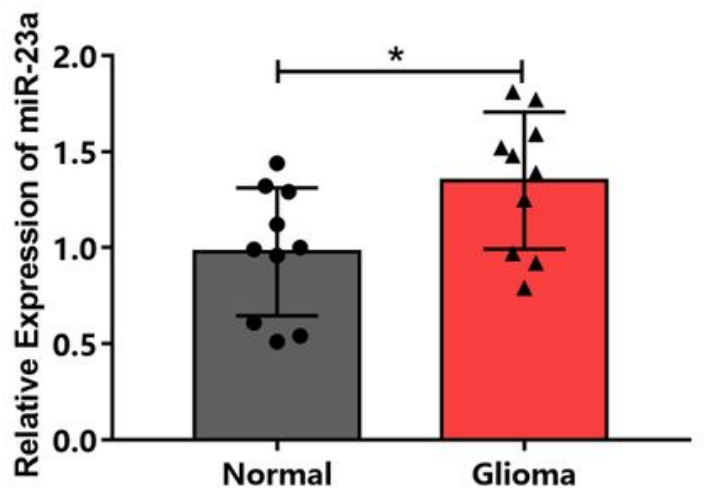

D

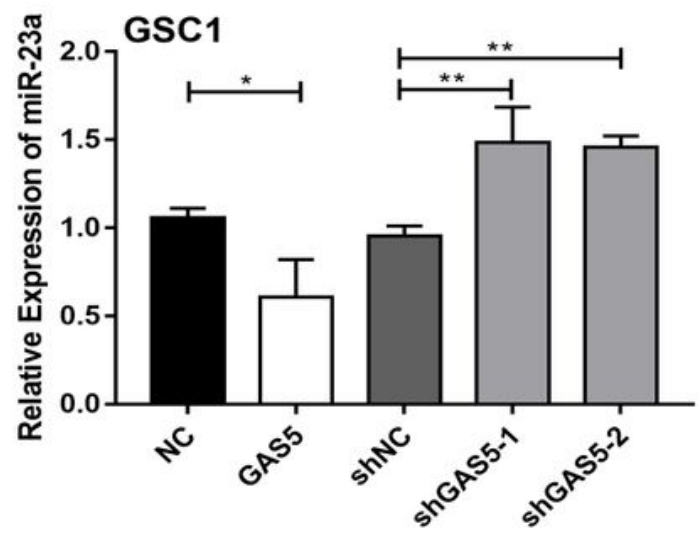

F

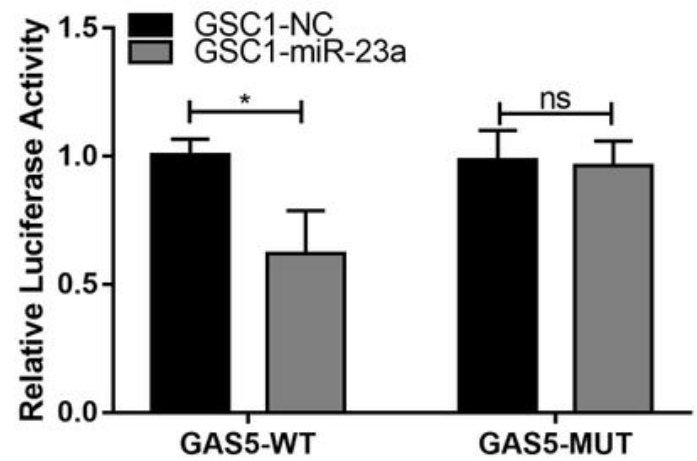

C

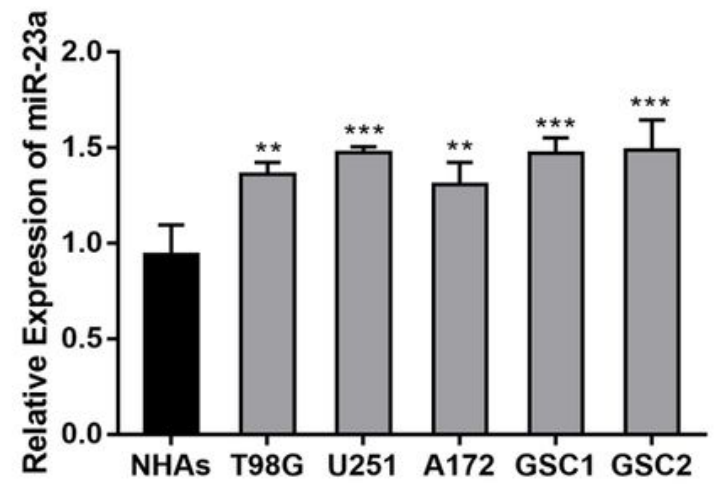

E

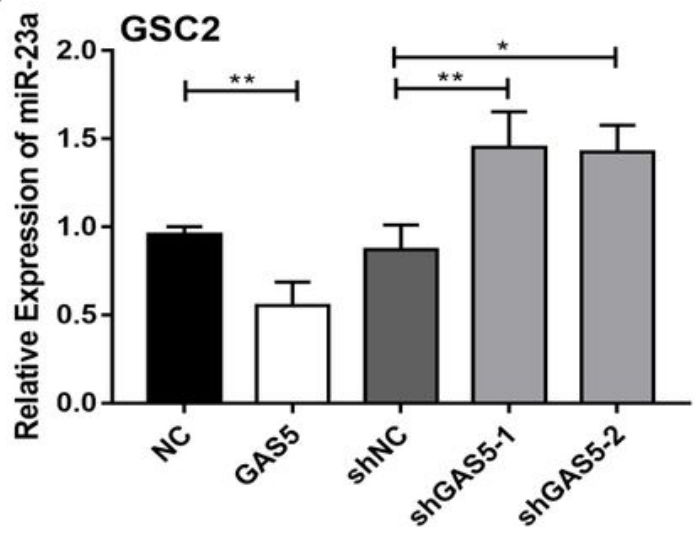

G

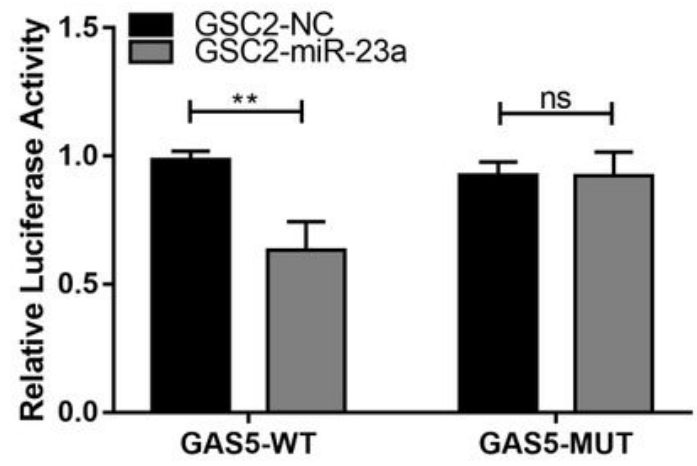

Figure 6

(A) the putative bonding site between miR-23a and GAS5. Wild type (WT) and mutant type (MUT) vector of GAS5 were constructed for luciferase activity assays. (B, C) MiR-23a expression level was evaluated by qRT-PCR in GSCs, glioma cell lines and glioma tissue. ${ }^{\star} p<0.05$, ${ }^{\star \star} p<0.01$, ${ }^{\star \star *} p<0.001$, one-way ANOVA. (D, E) qRT-PCR assays showed miR-23a was negatively regulated by GAS5 in GSCs. ${ }^{\star} p<0.05,{ }^{\star \star} p<0.01$, 
one-way ANOVA; Student's t test. (F, G) Luciferase reporter assays were performed to verify the bonding between miR-23a and GAS5. ${ }^{\star} p<0.05,{ }^{* \star} p<0.01$, Student's t test.

A

miR-23a 3'-ccuuuagggaccgUUACACUa-5'

CDH1 WT 5'-cucuuuuuauuaAAUUGUGAa-3'

CDH1 MUT 5'-cucuuuuuauuuaAAUGUGAa-3'
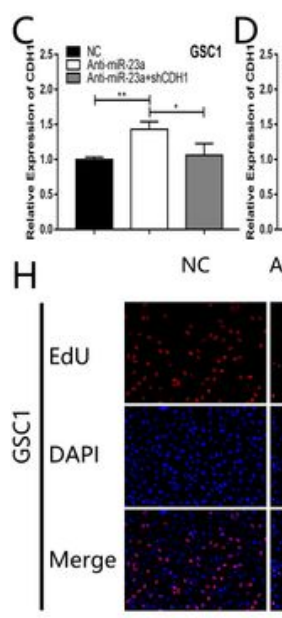

NC
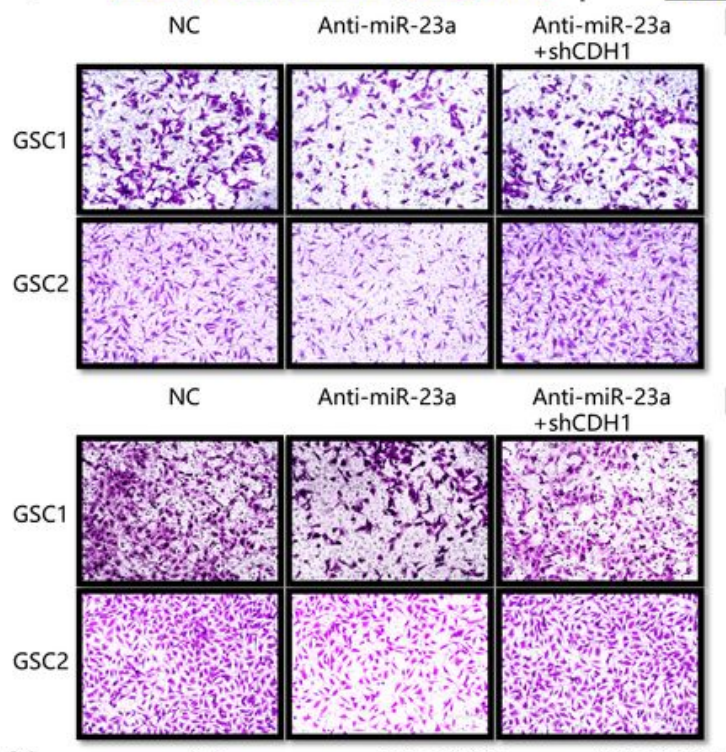

N
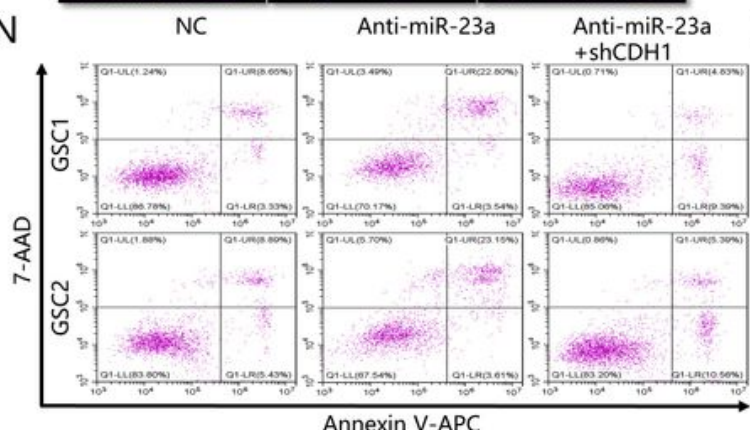

Annexin V-APC

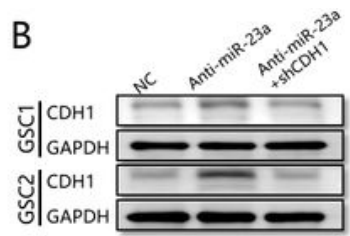

G

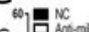

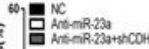
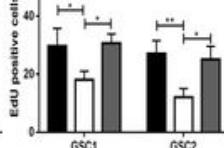

NC Anti-miR-23a Anti-miR-23a

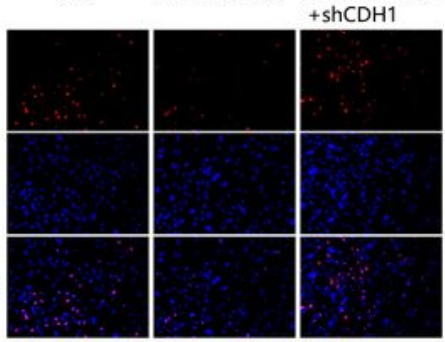

K
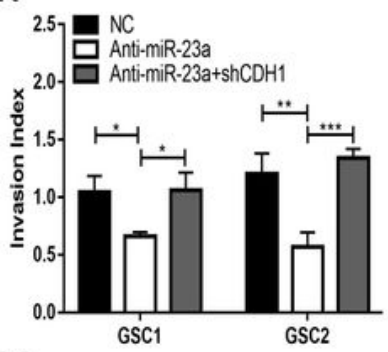

$\mathrm{M}_{2.5} \mathrm{MC}$

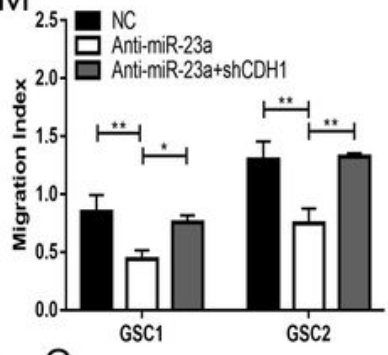

$\mathrm{O}$

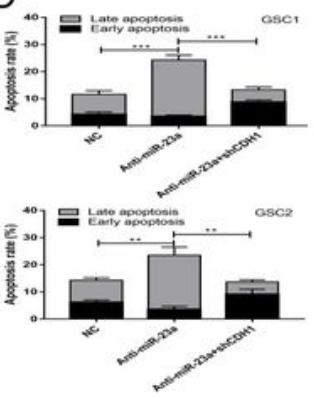

Figure 7

(A) The predicated bonding sites of miR-23a on CDH1. The vector of CDH-WT and CDH1-MUT were constructed for luciferase activity assays. (B-D) qRT-PCR and western blot analysis of CDH1 expression in 
GSCs transfected with miR-23a inhibitor or miR-23a inhibitor together with CDH1 shRNA. ${ }^{*} \mathrm{p}<0.05$, ${ }^{* \star} \mathrm{p}<$ 0.01 , one-way ANOVA. (E, F) Luciferase reporter assays testified that miR-23a weakened the luciferase activity of CDH1-WT in GSCs. * $p<0.05$, Student's $t$ test. (G-I) The proliferation capacity was analyzed in GSCs transfected with miR-23a inhibitor or miR-23a inhibitor together with CDH1 shRNA by EdU assays. ${ }^{*} \mathrm{p}<0.05$, ${ }^{*} \mathrm{p}<0.01$, one-way ANOVA. (J-M) The ability of invasion and migration was evaluated in GSCs transfected with miR-23a inhibitor or miR-23a inhibitor together with $\mathrm{CDH} 1$ shRNA via transwell assays. ${ }^{*} \mathrm{p}<0.05,{ }^{*} \mathrm{p}<0.01,{ }^{* \star} \mathrm{p}<0.001$, one-way ANOVA. $(\mathrm{N}-\mathrm{O})$ The cell apoptosis rate was determined in GSCs transfected with miR-23a inhibitor or miR-23a inhibitor together with CDH1 shRNA via flow cytometric assays. ${ }^{\star *} p<0.01,{ }^{\star *} p<0.001$, one-way ANOVA. 

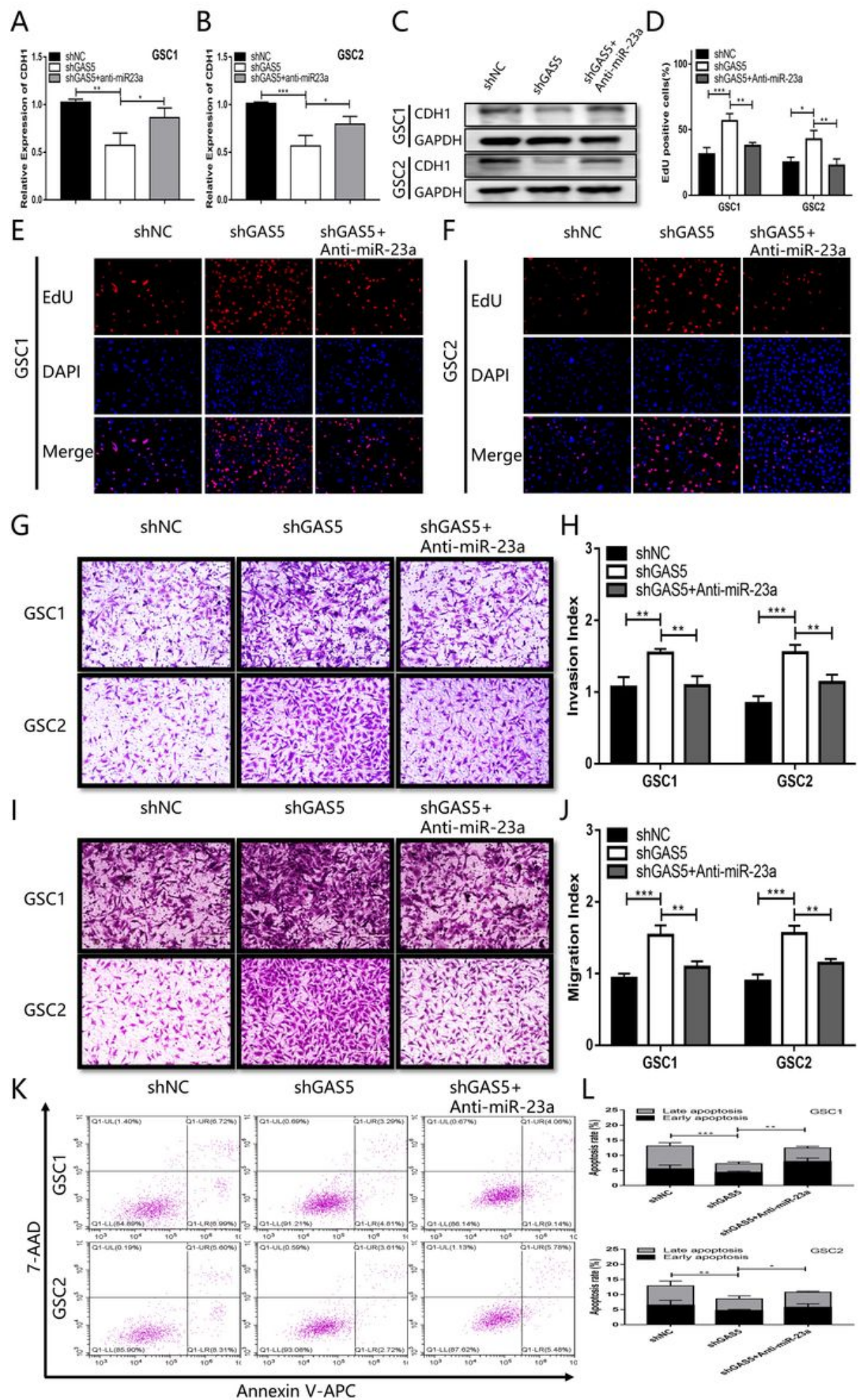

\section{Figure 8}

(A-C) qRT-PCR and western blot analysis of CDH1 expression in GSCs transfected with shGAS5 or shGAS5 together with miR-23a inhibitors. ${ }^{\star} p<0.05,{ }^{\star *} p<0.01,{ }^{* \star} p<0.001$, one-way ANOVA. (D-F) EdU assays were performed to analyze the proliferation ability of GSCs transfected with shGAS5 or shGAS5 together with miR-23a inhibitors. ${ }^{*} p<0.05,{ }^{*} p<<0.01,{ }^{* \star *} p<0.001$, one-way ANOVA. (G-J) The capacity of invasion and migration was evaluated in GSCs transfected with shGAS5 or shGAS5 together with miR- 
23a inhibitors by transwell assays. ${ }^{\star \star} p<0.01$, ${ }^{\star \star \star} p<0.001$, one-way ANOVA. $(K, L)$ Apoptosis rate was assessed in GSCs transfected with shGAS5 or shGAS5 together with miR-23a inhibitors by flow cytometric analysis. ${ }^{*} \mathrm{p}<0.05,{ }^{* \star} \mathrm{p}<0.01,{ }^{* \star *} \mathrm{p}<0.001$, one-way ANOVA. 